\title{
Ärzte, die mit gutem Beispiel vorangehen, sind überzeugender
}

Wie oft haben Sie Ihren Patienten schon geraten, ein paar Pfund abzunehmen, gesünder zu essen, nicht mehr zu rauchen? Und hatten Sie dabei das Gefühl, dass Ihr guter Rat in den Wind gesprochen war? Vielleicht kommt die Botschaft eher an, wenn Sie selbst mehr für Ihre Fitness tun.

Dies behaupten jedenfalls Forscher des University of Michigan Cardiovascular Center. Sie befragten Ärzte nach ihrem persönlichen Fitnessprogramm. Außer- dem wurde gefragt, ob die Kollegen ihre Patienten zu Sport anhielten und ob sie der Meinung seien, dass ihre Botschaft überzeugend ankomme. Je mehr die Ärzte für ihre eigene Fitness unternahmen, desto überzeugter waren sie, dass sie auch ihre Patienten zu einem gesünderen Lebensstil bewegen könnten. Ob die Patienten den Rat ihrer Ärzte tatsächlich befolgten, wurde nicht untersucht.

Preventive Cardiology, 2010; DOI: 10.1111/j.1751-7141.2010.00079.X

\section{ARTHROSE}

\section{Oraler Knorpelersatz wirkungslos}

Durch die Einnahme von Glucosamin und Chondroitin soll bei Arthrose der Knorpelverlust in den betroffenen Gelenken etwas ausgeglichen werden. Das Geschäft mit den entsprechenden Supplementen floriert. Allein für Glucosaminpräparate wurden im Jahr 2008 weltweit fast 800 Mio. Euro ausgegeben. Doch dieses Geld könnte man sich sparen, wenn sich eine Metaanalyse der Universität Bern bewahrheitet. Danach haben weder Glu-

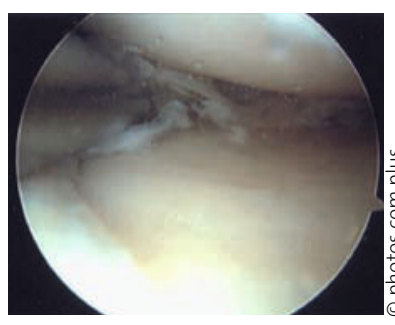
cosamin noch Chondroitin oder die Kombination einen klinisch relevanten Einfluss auf den Gelenkschmerz oder die Verengung des Gelenkspalts. In der Metaanalyse waren zehn Studien mit insgesamt 3803 Patienten ausgewertet worden. BMJ 2010;341:C4675

\section{PSYCHOLOGIE}

\section{Hör auf deine innere Stimme!}

Ob es nun das Freudsche Über-Ich ist oder einfach nur unser Gewissen, in unserem Köpfen spielt sich in allen möglichen Lebenslagen ein ständiger Dialog ab: „Iss das nicht", sagt die innere Stimme, wenn wir zu einem zweiten Stück Kuchen greifen wollen. Und haben wir bestimmte Aufgaben zu lösen, sagt uns die Stimme vor, wie es geht.

Forscher konnten jetzt zeigen, dass wir besser auf unsere innere Stimme hören sollten. Probanden mussten auf Signale reagieren und an einem Computer Knöpfe drücken oder eben nicht drücken. Im ersten Test durften sich die Probanden vorsagen „drücken“ oder „nicht drücken“. In einem zweiten Test wurde die innere Stimme unterdrückt, indem die Probanden ein bestimmtes Wort endlos wiederholen mussten. Die Fehlerquote ging prompt signifikant nach oben. Acta Psychologica 2010, im Druck

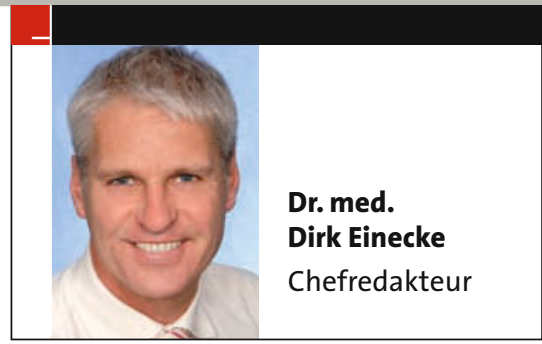

INFLUENZA-VAKZINE

\section{Impfung gegen Herzinfarkt}

KHK-Patienten sollen laut STIKO und laut Deutscher Gesellschaft für Kardiologie gegen Influenza geimpft werden. Gestützt wird die Empfehlung u.a. durch eine jetzt veröffentlichte Studie mit 16012 Herzinfarktpatienten und 62694 Kontrollpersonen: Personen, die innerhalb der letzten zwölf Monate geimpft worden waren, hatten ein um 19\% geringeres Risiko für einen akuten Myokardinfarkt als Personen ohne Impfschutz. Besonders ausgeprägt war der Schutzeffekt, wenn die Impfung bereits vor Mitte November erfolgt war. Eine Impfung gegen Pneumokokken hatte in derselben Studie keine Auswirkung auf das Herzinfarktrisiko. CMAJ 2010;DOI:10.1503/cmaj.091891

\section{KOMPRESSIONSSTRÜMPFE}

\section{Knielang oder bis zum Oberschenkel?}

Kompressionsstrümpfe, die bis zum Oberschenkel reichen, verhindern 30\% mehr tiefe Beinvenenthrombosen als knielange Strümpfe. Dies ergab die CLOT-Studie, für die 3000 bettlägerige Schlaganfallpatienten rekrutiert wurden. Insgesamt waren aber weder die langen noch die kürzeren Kompressionsstrümpfe besonders effektiv in der Thromboseprophylaxe nach Schlaganfall. Das National Institute for Clinical Excellence hat in Anbetracht dieser Ergebnisse die Leitlinien geändert und empfiehlt für Schlaganfallpatienten gar keine Kompressionsstrümpfe mehr.

Pressemitteilung der University of Edinburgh, 9/2010 